\title{
A comparison of three different surgery approaches and methods for neurologically intact thoracolumbar fractures: A retrospective study
}

Chao Zhu ( $\sim$ zhuchao3808@sina.com )

nanjing jiangning hospital

bin wang

The affiliated jiangning hospital with nanjing medical university

jian yin

The affiliated jiangning hospital with nanjing medical university

Xinhui Liu

The affiliated jiangning hospital with nanjing medical university

\section{Research Article}

Keywords: pedicle screw fixation, open, percutaneous, paraspinal, thoracolumbar fracture

Posted Date: March 24th, 2021

DOI: https://doi.org/10.21203/rs.3.rs-289274/v1

License: (c) (i) This work is licensed under a Creative Commons Attribution 4.0 International License.

Read Full License 


\section{Abstract \\ Objectives}

The purpose of this study was to evaluate and compare the feasibility, safety, and efficacy of conventional open pedicle screw fixation (COPSF), percutaneous pedicle screw fixation (PPSF), and paraspinal posterior open approach pedicle screw fixation (POPSF) for treating neurologically intact thoracolumbar fractures.

\section{Methods}

We retrospectively reviewed 108 patients who were posteriorly stabilized without graft fusion. Among them, 36 patients underwent COPSF, 38 patients underwent PPSF, and 34 patients underwent POPSF. The clinical outcomes, relative operation indexes, radiological findings were assessed and compared among the 3 groups.

\section{Results}

All of the patients were followed up for a mean time of 20 months. The PPSF group and POPSF group had shorter operation times, lower amounts of intraoperative blood loss, and shorter postoperative hospital stays than the COPSF group $(P<0.05)$. The radiation times and hospitalization costs were highest in the PPSF group $(P<0.05)$. Every group exhibited significant improvements in the Cobb angle $(C A)$ and the vertebral body angle (VBA) correction (all $P<0.05$ ). The COPSF group and the POPSF group had better improvements than the PPSF group at 3 days postoperatio and the POPSF group had the best improvements in the last follow up $(P<0.05)$.

\section{Conclusion}

Both PPSF and POPSF achieved similar effects as COPSF while also resulting in lower incidences of injury. PPSF is more advantageous in the early rehabilitation time period, compared with COPSF, but POPSF is a better option when considering the long-term effects, the costs of treatment and the radiation times.

\section{Introducton}

Most spinal fractures occur in the thoracolumbar segment, which is biomechanically weak against external injury ${ }^{[1,2]}$. Although the management of thoracolumbar fractures remains controversial ${ }^{[3]}$, it has been proven that surgical treatment can often achieve better clinical outcomes than conservative management ${ }^{[4]}$. Short-segment pedicle spinal instrumentation leads to the correction of kyphotic 
deformities, a greater initial stability, and early painless mobilization ${ }^{[5,6]}$. However, a conventional open approach can result in some disadvantages, including blood loss, long durations of hospital stay, and injury to the paraspinal muscles ${ }^{[7,8]}$.

In 1968, Wiltse first reported the paraspinal posterior open approach pedicle screw fixation (POPSF) method as a minimally invasive approach for treating lumbar spinal fractures ${ }^{[0]}$. Studies have proven that the minimally invasive approach is superior to the conventional open approach, in terms of reduced muscle injuries.

In recent years, with the rapid development of modern navigation devices, percutaneous pedicle screw fixation (PPSF) has been widely used in spinal surgery as a minimally invasive technique from the time at which it was first reported by Magerl in $1984^{[10-12]}$.

A number of clinical studies have compared PPSF to conventional open approach pedicle screw fixation $(\mathrm{COPSF})^{[13-15]}$. Some clinical studies have also compared the paraspinal posterior open approach pedicle screw fixation (POPSF) to COPSF in the treatment of thoracolumbar fractures ${ }^{[16,17]}$. However, few studies have compared these three approaches/methods at the same time. In this study, we compared related data from patients with neurologically intact thoracolumbar fractures who had undergone shortsegment pedicle fixation, either by the conventional posterior open approach or by the minimally invasive approach containing PPSF and POPSF. The purpose of this study was to provide a scientific basis for the suitable choice of the surgical approaches for patients with thoracolumbar fractures.

\section{Materials And Methods}

\section{Patient samples}

We retrospectively reviewed 108 cases of single-segment, neurologically intact thoracolumbar (T11-L2) fractures from January 2012 to August 2016. All of the patients were treated in the Orthopaedics Department of our hospital. The fracture type was classified as Type A according to the new AO thoracolumbar fracture classification system ${ }^{[18]}$. All of the patients received operations within two weeks following the injury. The indications for operation were as follows: $a$. Type $A 1$ and $C A>15^{\circ}, b$. dynamic fracture, c. kyphotic deformity getting larger after admission. These patients were divided into 3 groups based on the surgical approach and method in this study: a COPSF group (36 patients who received conventional open approach pedicle screw fixation), a PPSF group (38 patients who underwent percutaneous pedicle screw fixation), and a POPSF group (34 patients who were treated with paraspinal posterior open approach pedicle screw fixation). Before the surgery, we introduced the characteristics of three surgical approachs and methods to the patients and their family. According to patients' conditions and permissions, we performed operations by different surgical approachs and methods. All operations were performed by the same group of doctors, and the chief surgeon was the corresponding author. All of the patients received a 4-pedicle screw fixation. We did not analyse patients who received a pedicle screw in the fractured vertebra. Procedures were performed in accordance with the Declaration of Helsinki and 
were approved by the Ethics Committee of Human Experimentation of our hospital. The prove reg. number was 2011030. All of the patients signed corresponding informed consents before the study. The exclusion criteria were as follows: 1) pregnancy or pathologic and osteoporotic fractures, 2) younger than 18-years-old or old than 60-years-old, 3 ) an earlier surgery had been performed at the fracture site, and 4) the initial fractures were combined with other diseases that could significantly influence daily life.

\section{Reduction system}

In the COPSF group and POPSF group, the EXPEDIUM Spine System (DePuy Synthes, Raynham, Massachusetts, USA) was used, however, the VIPER MIS Spine System (DePuy Synthes, Raynham, Massachusetts, USA) was used in the PPSF group. All the pedicles in three groups were monoaxial. All the instrumentations in three groups were routinely removed 12 to 18 months after operation.

\section{Surgical procedure}

\section{PPSF group}

After the general anesthesia but before the operation, the patient was place in a prone position for several minutes and the kyphosis of the injured vertebral body was partly corrected by hyperextension. We used a preoperative locator to aid in locating the pedicle projection ${ }^{[19]}$. The preoperative locator was made of stainless steel. The locator consisted of several horizontal and longitudinal rods. Different marks were made on the rods, and there were $1-\mathrm{cm}$ spaces between each horizontal rod. The patient was placed into a prone position after receiving general endotracheal anaesthesia, and silicone pads were used to support the chest, abdomen, and pelvis. The preoperative locator was placed on the back of each of the patients' back with the central part (Fig. 1A). The correct pedicle projection and incision were obtained according to the different markers on the locator after observing the AP fluoroscopic image (Fig. 1B). An approximately $1.5-\mathrm{cm}$ incision was performed, and the underlying fascia was bluntly dissected. A puncture catheter was positioned on the outer and lower edges of the pedicle and was slowly advanced into the pedicle and posterior half of the vertebral body. The guide wire was then inserted into the catheter, and the needle was carefully removed (Fig. 1C\&D). The fascia and soft tissue were separated by using a series of sequential dilators. A self-tapping, cannulated pedicle screw with an appropriate length and diameter was inserted into the vertebra through the guide wire under the protection of the outside catheter (Fig. 1E), and then, the rod was installed (Fig. 1F). The rods were fixed using screws. At first, we only tightened the screws at one end, and then lever of the screw towers of the other end to generate additional lordosis to correct the kyphosis. After the reduction, all the screws were tightened.

All of the procedures were performed with the use of G-arm fluoroscopic image guidance.

\section{COPSF group}

The patients in the COPSF group were treated with conventional open pedicle screw fixation surgery according to the previous study. ${ }^{7}$ The reduction technique was the same as PPSF group. 


\section{POPSF group}

The positions of the fractured vertebrae were ascertained by using the G-arm. After routine sterilization and placement of the drapes, an approximate 8- to 10-cm midline incision was performed in the target segment. Subsequently, the thoracolumbar fascia, multifidus, and longissimus were separated in order to reach the pedicle entry point. The next procedure was the same as the traditional method (Fig. 2).

\section{Observation Index}

Three groups were compared in terms of operating time, intraoperative blood loss, intraoperative fluoroscopy, hospital stay, hospitalization cost and postoperative complications. The vertebral body angle (VBA) and Cobb's angle (CA) were evaluated at pre-operation, the third day after surgery, and the final follow-up. The VBA and CA were measured by conventional radiograph (X-ray). The visual analog scores (VAS) was evaluated at pre-operation, the seventh day after surgery, and the final follow-up. Oswestry disability index (ODI) scores were evaluated at pre-operation and the final follow-up. Levels of serum creatine kinase (CK) were measured at pre-operation, 1 day and 1 week after the operation.

\section{Statistical analysis}

All of the statistical analyses in this study were performed by using SPSS 17.0 statistical software (IL, USA). The variables with continuous data were reported as means and standard deviations. Statistical analyses were conducted by using one-way ANOVA to compare the means if they were accord with normal distribution. Where the normal distribution was not met, the Wilcoxon rank sum test was used. The categorical variables that were demonstrated as counted data were compared by using the $\chi^{2}$ test. $P$ $<0.05$ indicated statistical significance.

\section{Results}

The demographic and clinical characteristics of a total of 108 patients, including 68 males and 40 females (mean age: 43.1 years; range: $24-60$ years), are shown in Table 1. Injuries were due to falling from a high height in 55 patients, traffic accidents in 30 patients, and falling from a low height in 23 patients. There were 36 patients in COPSF group, 38 patients in PPSF group and 34 patients in POPSF group. In the COPSF group, 15 for type A1, 3 for type A2, 10 for type A3 and 8 for type A4. In the PPSF group, 16 for type A1, 4 for type A2, 13 for type A3 and 15 for type A4. In the POPSF group, 15 for type A1, 2 for type $A 2,10$ for type $A 3$ and 7 for type A4. No patient required a posterior decompression of the vertebral canal, according to CT and MRI scans. There were no significant differences in mean age, body mass index, gender, fracture level, and fracture classification among the COPSF group, the PPSF group and the POPSF group (all $P>0.05$, Table 1). All of the patients were followed up for a mean time of 20 months (ranging from 14 to 38 months).

Table 1 Comparison of the general data among the three groups 


\begin{tabular}{|lllll|}
\hline Characteristics & $\begin{array}{l}\text { COPSF } \\
\text { group }\end{array}$ & $\begin{array}{l}\text { PPSF } \\
\text { group }\end{array}$ & $\begin{array}{l}\text { POPSF } \\
\text { group }\end{array}$ & P value \\
\hline Cases (n) & 36 & 38 & 34 & \\
\hline Mean age (y) & $46.3 \pm 7.8$ & $47.9 \pm 8.5$ & $45.6 \pm 6.8$ & $0.80^{*}$ \\
\hline Gender & & & & \\
\hline Male & 21 & 25 & 22 & $0.78^{\Delta}$ \\
\hline Female & 15 & 13 & 12 & \\
\hline BMI & $22.0 \pm 2.5$ & $21.8 \pm 2.4$ & $22.1 \pm 2.6$ & $0.87^{*}$ \\
\hline Fracture level & & & & \\
\hline T11 & 6 & 8 & 4 & $0.93^{\Delta}$ \\
\hline T12 & 13 & 13 & 10 & \\
\hline L1 & 11 & 12 & 13 & \\
\hline L2 & 6 & 6 & 7 & \\
\hline Fracture classification & & & & \\
\hline A1 & 15 & 16 & 15 & $0.94^{\Delta}$ \\
\hline A2 & 3 & 4 & 2 & \\
\hline A3 & 10 & 13 & 10 & \\
\hline A4 & 8 & 5 & 7 & \\
\hline
\end{tabular}

*one-way ANOVA.

${ }^{\Delta} \chi^{2}$ test.

COPSF: conventional open approach pedicle screw fixation

PPSF: percutaneous pedicle screw fixation

POPSF: posterior open approach pedicle screw fixation

\section{Operation indexes}

PPSF and POPSF resulted in reduced injuries, including shorter operation times, lower amounts of intraoperative blood loss, and shorter postoperative hospital stays, compared with the COPSF (Table 2). 
The PPSF group had the least amounts of intraoperative blood loss $(P<0.05)$. The intraoperative radiation times and hospitalization costs were highest in the PPSF group $(P<0.05)$, but there were no significant differences between the POPSF group and the COPSF group $(P>0.05)$. There were no significant differences among the 3 groups in terms of complications, as shown in Table $3(P>0.05)$.

Table 2 Comparison of operation indexes among the three groups.

\begin{tabular}{|llll|}
\hline Operation indexes & COPSF group & PPSF group & POPSF group \\
\hline Operating time (min) & $125.3 \pm 23.4$ & $98.4 \pm 25.3^{*}$ & $97.6 \pm 19.6^{\Delta}$ \\
\hline Intraoperative blood loss $₫ \mathrm{ml} \rrbracket$ & $367.9 \pm 37.6$ & $107.9 \pm 18.7^{*}$ & $140.1 \pm 25.8^{\Delta \#}$ \\
\hline Intraoperative fluoroscopy (times) & $3.3 \pm 1.1$ & $28.4 \pm 1.4^{*}$ & $3.7 \pm 1.3^{\#}$ \\
\hline Hospital stay $\llbracket \mathrm{d} \mathbb{}$ & $14.4 \pm 1.8$ & $10.1 \pm 2.1^{*}$ & $11.2 \pm 2.8^{\Delta}$ \\
\hline Hospitalization cost $\left(\times 10^{3}\right.$ & $49.9 \pm 0.9$ & $55.2 \pm 1.0^{*}$ & $48.6 \pm 1.3^{\#}$ \\
$\mathrm{CNY})$ & & & \\
\hline
\end{tabular}

*Significant difference between the PPSF and the COPSF groups.

${ }^{\Delta}$ Significant difference between the POPSF and the COPSF groups.

\# Significant difference between the POPSF and the PPSF groups.

CNY indicates China Yuan.

One-way ANOVA was used to compare the operating times and the hospitalization costs.

Wilcoxon rank sum tests were used to compare the intraoperative blood loss, intraoperative fluoroscopy, and hospital stays.

Table 3 Comparison of the postoperative complications among the three groups.

\begin{tabular}{|lllll|}
\hline Postoperative complications & COPSF group & PPSF group & $\begin{array}{l}\text { POPSF } \\
\text { group }\end{array}$ & P value $^{\star}$ \\
\hline Incorrect screw positioning & 1 & 2 & 2 & 0.82 \\
\hline Incision infection & 1 & 0 & 0 & 0.36 \\
\hline Neurological symptom & 1 & 2 & 1 & 0.82 \\
\hline
\end{tabular}

${ }^{*} \chi^{2}$ test 


\section{Radiological Results}

Each group exhibited significant improvements in the Cobb angle (CA) and the vertebral body angle (VBA) correction (all $\mathrm{P}<0.05$ ). The COPSF group and the POPSF group had better improvements in both CA and VBA than the PPSF group at 3-days post operation. No significant differences were observed between the COPSF group and the PPSF group in the last follow-up $(P>0.05)$, whereas the POPSF group had the best improvement in the last follow-up $(P<0.05)$ (Table 4$)$.

Table 4 Comparison of radiological parameters among the three groups.

\begin{tabular}{|llll|}
\hline Radiological parameters & COPSF group & PPSF group & POPSF group \\
\hline CA $\left(^{\circ}\right)$ & & & \\
\hline Preoperative & $17.4 \pm 5.4$ & $17.3 \pm 5.1$ & $18.1 \pm 5.8$ \\
\hline 3 d post & $4.1 \pm 1.7$ & $5.7 \pm 2.4^{\star}$ & $4.3 \pm 2.2^{\#}$ \\
\hline Last & $7.6 \pm 2.8$ & $7.9 \pm 2.7$ & $5.9 \pm 2.9^{\triangle \#}$ \\
\hline VBA $\left(^{\circ}\right)$ & & & \\
\hline Preoperative & $20.9 \pm 5.0$ & $21.2 \pm 5.5$ & $21.9 \pm 6.1$ \\
\hline 3 d post & $7.0 \pm 3.0$ & $9.1 \pm 3.2^{*}$ & $7.2 \pm 3.3^{\#}$ \\
\hline Last & $9.3 \pm 3.2$ & $10.6 \pm 3.5$ & $8.3 \pm 4.0^{\#}$ \\
\hline
\end{tabular}

*Significant difference between the PPSF and the COPSF groups.

${ }^{\Delta}$ Significant difference between the POPSF and the COPSF groups.

\# Significant difference between the POPSF and the PPSF groups.

CA: Cobb angle, VBA: vertebral body angle.

Using one-way ANOVA.

\section{Effectiveness parameters}

All of the groups exhibited significant reductions from baseline in the mean visual analogue scores (VAS) after the operation. There were significant differences in the VAS at 7 days after surgery among the three groups $(P<0.05)$, and it was the lowest in the PPSF group. At the last follow up, there were no significant 
differences in the VAS and the Oswestry disability index (ODI) score between the PPSF group and the POPSF group. However, there were significant differences in the VAS and ODI score between these two groups and the COPSF group $(P<0.05)$, and both the VAS and ODI score were the highest in the COPSF group (Table 5).

Table 5 Comparison of pain assessment and function evaluation among the three groups.

\begin{tabular}{|llll|}
\hline Clinical parameters & COPSF group & PPSF group & POPSF group \\
\hline VAS & & & \\
\hline Preoperative & $6.8 \pm 1.3$ & $6.7 \pm 0.9$ & $7.0 \pm 1.1$ \\
\hline 7 days post & $3.3 \pm 1.1$ & $2.2 \pm 0.8^{\star}$ & $2.7 \pm 0.8^{\Delta \#}$ \\
\hline Last follow up & $1.3 \pm 0.5$ & $0.7 \pm 0.5^{\star}$ & $0.8 \pm 0.6^{\Delta}$ \\
\hline ODI & & & \\
\hline Preoperative & $90.6 \pm 3.1$ & $90.3 \pm 2.5$ & $90.9 \pm 2.6^{\star}$ \\
\hline Last follow up & $6.2 \pm 2.2$ & $3.2 \pm 2.1^{\star}$ & $4.1 \pm 1.9^{\Delta}$ \\
\hline
\end{tabular}

*Significant difference between the PPSF and the COPSF groups.

${ }^{\triangle}$ Significant difference between the POPSF and the COPSF groups.

\# Significant difference between the POPSF and the PPSF groups.

VAS: visual analogue scale, ODI: Oswestry disability index.

Using one-way ANOVA.

\section{Laboratory parameters}

Creatine kinase (CK) is released into the blood when skeletal muscle cells are damaged; thus, the content of serum CK levels can indicate the level of muscle damage ${ }^{[20]}$. All of the groups exhibited significant increases in the CK levels after the operation. There were significant differences in the CK levels at 1 day after surgery among the three groups $(P<0.05)$. The CK levels were the highest in the COPSF group and the lowest in the PPSF group. There were no significant differences in the CK levels at 7 days after surgery among the three groups $(P>0.05)$ (Table 6).

Table 6 Comparison of the serum creatine kinase levels among the three groups. 


\begin{tabular}{|llll|}
\hline $\begin{array}{l}\text { Laboratory } \\
\text { parameters }\end{array}$ & COPSF group & PPSF group & POPSF group \\
\hline serum CK $(\mathrm{U} / \mathrm{L})$ & & & \\
\hline Preoperative & $215.1 \pm 68.9$ & $223.2 \pm 55.4$ & $205.4 \pm 50.6$ \\
\hline 1 d post & $952.6 \pm 379.1$ & $484.3 \pm 234.7^{\star}$ & $690.0 \pm 232.9^{\Delta \#}$ \\
\hline 1 week post & $185.5 \pm 59.6$ & $155.4 \pm 55.7$ & $177.5 \pm 59.1$ \\
\hline
\end{tabular}

*Significant difference between the PPSF and the COPSF groups.

${ }^{\Delta}$ Significant difference between the POPSF and the COPSF groups.

\# Significant difference between the POPSF and the PPSF groups.

serum CK: serum creatine kinase.

Using one-way ANOVA.

\section{Discussion}

The treatment of neurologically intact thoracolumbar fractures is still unclear ${ }^{[21,22]}$. Conservative treatment has achieved satisfactory outcomes in several neurologically intact thoracolumbar fracture cases while avoiding surgical complications ${ }^{[23]}$. Most scholars believe that surgical treatment can not only correct kyphotic deformities, reduce pain, and allow patients to return to daily life activities at an earlier time but can also aid in avoiding the occurrence of delayed kyphosis and neurological symptoms $^{[24,25]}$.

The conventional open posterior pedicle screw fixation method has been widely used for the treatment of neurologically intact thoracolumbar fractures. This approach has a clear exposure to the vertebrae and a shorter learning curve. However, it is also associated with higher infection rates, prolonged operation times, greater amounts of blood loss, and longer hospital stays ${ }^{[26]}$.

Percutaneous pedicle screw fixation is classified as a minimally invasive surgery that does not require the need to peel the paraspinal muscles during the operation, thus reducing the chance of injury. Patients can recover more quickly after surgery ${ }^{[27]}$. In this study, operation times, amounts of blood loss, VAS scores, and hospital stays were significantly lower in the PPSF group than those in the COPSF group, which is consistent with previous studies ${ }^{[13-15]}$.

Paraspinal posterior open approach pedicle screw fixation is also classified as a minimally invasive surgery. The blunt dissection from the interspace between the multifidus and longissimus can significantly reduce injury to the muscle ${ }^{[17]}$. In the POPSF group, operation times, amounts of blood loss, 
VAS scores, and hospital stays were also significantly lower than those in the COPSF group. After the muscles are damaged, creatine kinase in the muscle cells is released into the blood. Therefore, the content of serum creatine kinase levels can be used as an indicator of muscle injury ${ }^{[28]}$. Our results showed that serum creatine kinase levels were significantly lower in the PPSF group and in the POPSF group than those in the COPSF group on postoperative day 1 and was the lowest amount in this group as well. VAS and hospital stays were significantly lower in the PPSF group than those in the POPSF group. These findings suggested that although both the PPSF group and the POPSF group are classified as minimally invasive approaches, percutaneous surgery is more advantageous in early recovery than paraspinal approach surgery. Our study showed that the operation time of the PPSF group was significantly higher than that of both the COPSF group and the POPSF group, and there was no significant difference between the COPSF group and the POPSF group. Similar results were observed when comparing G-arm exposure times. The surgeons and patients received higher doses of radiation in the PPSF group. Due to the price of the implanted device, the hospitalization costs in the PPSF group were higher than those of the COPSF and POPSF groups.

It has been reported that deviations of approximately $3 \%$ in the accuracy rates of percutaneous pedicle screw insertions are unacceptable ${ }^{[29]}$. Our accuracy results were $99.3 \%(143 / 144)$ in the COPSF group, $98.7 \%$ (150/152) in the PPSF group, and 98.5\% (134/136) in the POPSF group, and all of the complications exhibited no significant differences among the 3 groups. Although the operation of implantation of pedicle screws was not performed under direct vision, the accuracy was guaranteed, due to the use of repeated fluoroscopy during the procedure. Our results were similar to those of some other studies $^{[13,30]}$.

Our radiological results indicated that the pre-operative and postoperative Cobb's angles and VBA exhibited significant differences in all three groups. In the PPSF group, the Cobb's angles and VBA improvements were less than those in the COPSF and POPSF groups, and no difference was found between the COPSF group and the POPSF group. Several studies have reported that, compared with mono-axial screws used in open surgery, percutaneous multiaxial screws are relatively weak in the bonescrew load, thus affecting the reduction effect ${ }^{[12,14]}$. However, as the percutaneous minimally invasive technique reduced the injury to the paraspinal muscle, the integrity of the ligamental structures was preserved; thus, the loss of the reduction was reduced ${ }^{[29,31]}$. There was no significant difference between the PPSF group and the COPSF group in the last follow-up, which showed that the Cobb angle correction and the VBA correction in the PPSF group were less than those in the COPSF group. The degree of paraspinal tissue injury through the use of the paraspinal posterior open approach was also lower, and the reduction loss was also lower. Our studies show that the long-term results for kyphosis correction in the POPSF group were best among the three groups.

Studies have shown that after lumbar muscle injury, the infiltration of adipose tissue can affect muscle contraction, thus leading to the recurrence of postoperative lower back pain (LBP) ${ }^{[32]}$. Our study showed that postoperative VAS scores were lower in the PPSF group than those in the POPSF group, but there 
was no difference in the long-term observation. In the final follow-up, VAS and ODI scores were highest in the open group, and we speculated that the recurrence of LBP in many patients of the COPSF was associated with muscle injury.

\section{Limitations}

Our study has some limitations. First, it was a single-center study with small sample size. Because the numbers of patients with type $\mathrm{A} 2, \mathrm{~A} 3$ and $\mathrm{A} 4$ thoracolumbar fractures were very small, we could not perform further comparative among different subtypes. Second, this study was a retrospective cohort study, so the cases were easily lost to follow-up. The last, we didn't use MRI, histological and electrophysiological analyses to assess multifidus muscle damages.

\section{Conclusions}

In conclusion, percutaneous pedicle screw fixation and paraspinal posterior open approach pedicle screw fixation are both acceptable, minimally invasive surgical-therapeutic choices for patients with neurologically intact thoracolumbar fractures. These techniques have a lot of advantages over conventional open posterior pedicle screw fixations. Percutaneous screws appear to be more advantageous in early rehabilitation time periods, but paraspinal posterior open approach pedicle screw fixation is a better option, given the long-term effects, the costs of treatment, and the amount of radiation doctors and patients receive.

\section{Abbreviations}

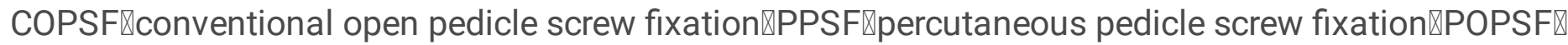

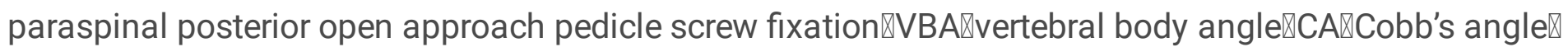

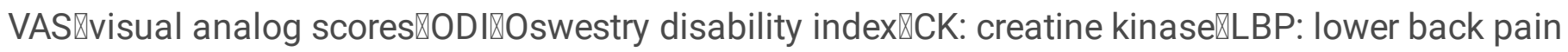

\section{Declarations}

\section{Acknowledgements:}

We appreciate the contribution of all patients, their families, the investigators, and the nursing staff from the Department of Orthopaedic Surgery, The Affiliated Jiangning Hospital with Nanjing Medical University.

\section{Authors' contributions:}

Xinhui Liu designed the study. Xinhui Liu, Chao Zhu, Bin Wang and Jin Yin performed all operations, Xinhui Liu was the chief surgeon. Chao Zhu and Bin Wang analyzed and interpreted the data. Chao Zhu and Jin Yin wrote the manuscript and Xinhui Liu approved the final version of the manuscript. All authors read and approved the final manuscript. 


\section{Funding}

There is no funding received.

\section{Availability of data and materials}

The data and materials contributing to this article may be made available upon request by sending an email to the correspondence author.

\section{Ethics approval and consent to participate}

The ethics committee of our hospital approved this study (NO: 2011030), and all the participants had written the informed consent.

\section{Consent for publication}

Written informed consent was obtained from each patient to authorize the publication of their data.

\section{Competing interests}

The authors declare no conflict of interests

\section{Author details}

Department of Orthopaedic Surgery, The Affiliated Jiangning Hospital with Nanjing Medical University, No. 169 Hushan Road, Nanjing 211100, Jiangsu Province, PR China.

\section{References}

1. Alpantaki K, Bano A, Pasku D, Mavrogenis AF, Papagelopoulos PJ, Sapkas GS, et al. Thoracolumbar burst fractures: a systematic review of management. Orthopedics. 2010;33(6):422-9. doi:10.3928/01477447-20100429-24.

2. Leucht P, Fischer K, Muhr G, Mueller EJ. Epidemiology of traumatic spine fractures. Injury. 2009;40(2):166-72. doi:10.1016/j.injury.2008.06.040.

3. Bohlman HH, Kirkpatrick JS, Delamarter RB, Leventhal M. Anterior decompression for late pain and paralysis after fractures of the thoracolumbar spine. Clinical orthopaedics and related research. 1994(300):24-9.

4. Abudou $M$, Chen $X$, Kong $X$, Wu T. Surgical versus non-surgical treatment for thoracolumbar burst fractures without neurological deficit. Cochrane Database Syst Rev. 2013(6):CD005079. doi:10.1002/14651858.CD005079.pub3.

5. Shen WJ, Liu TJ, Shen YS. Nonoperative treatment versus posterior fixation for thoracolumbar junction burst fractures without neurologic deficit. Spine. 2001;26(9):1038-45. doi:10.1097/00007632-200105010-00010. 
6. Fennell VS, Palejwala S, Skoch J, Stidd DA, Baaj AA. Freehand thoracic pedicle screw technique using a uniform entry point and sagittal trajectory for all levels: preliminary clinical experience. Journal of neurosurgery Spine. 2014;21(5):778-84. doi:10.3171/2014.7.SPINE1489.

7. Kim DY, Lee SH, Chung SK, Lee HY. Comparison of multifidus muscle atrophy and trunk extension muscle strength: percutaneous versus open pedicle screw fixation. Spine. 2005;30(1):123-9.

8. Mobbs RJ, Sivabalan P, Li J. Technique, challenges and indications for percutaneous pedicle screw fixation. Journal of clinical neuroscience: official journal of the Neurosurgical Society of Australasia. 2011;18(6):741-9. doi:10.1016/j.jocn.2010.09.019.

9. Wiltse LL, Bateman JG, Hutchinson RH, Nelson WE. The paraspinal sacrospinalis-splitting approach to the lumbar spine. The Journal of bone joint surgery American volume. 1968;50(5):919-26.

10. Magerl FP. Stabilization of the lower thoracic and lumbar spine with external skeletal fixation. Clinical orthopaedics and related research. 1984(189):125-41.

11. Kim BG, Dan JM, Shin DE. Treatment of thoracolumbar fracture. Asian spine journal. 2015;9(1):13346. doi:10.4184/asj.2015.9.1.133.

12. Wang H, Zhou Y, Li C, Liu J, Xiang L. Comparison of Open Versus Percutaneous Pedicle Screw Fixation Using the Sextant System in the Treatment of Traumatic Thoracolumbar Fractures. Clinical spine surgery. 2017;30(3):E239-E46. doi:10.1097/BSD.0000000000000135.

13. Wang B, Fan Y, Dong J, Wang H, Wang F, Liu Z, et al. A retrospective study comparing percutaneous and open pedicle screw fixation for thoracolumbar fractures with spinal injuries. Medicine. 2017;96(38):e8104. doi:10.1097/MD.0000000000008104.

14. Yang M, Zhao Q, Hao D, Chang Z, Liu S, Yin X. Comparison of clinical results between novel percutaneous pedicle screw and traditional open pedicle screw fixation for thoracolumbar fractures without neurological deficit. International orthopaedics. 2019;43(7):1749-54. doi:10.1007/s00264018-4012-x.

15. Kocis J, Kelbl M, Kocis T, Navrat T. Percutaneous versus open pedicle screw fixation for treatment of type $A$ thoracolumbar fractures. European journal of trauma emergency surgery: official publication of the European Trauma Society. 2020;46(1):147-52. doi:10.1007/s00068-018-0998-4.

16. Fan Y, Zhang J, He X, Huang Y, Wu Q, Hao D. A Comparison of the Mini-Open Wiltse Approach with Pedicle Screw Fixation and the Percutaneous Pedicle Screw Fixation for Neurologically Intact Thoracolumbar Fractures. Medical science monitor: international medical journal of experimental clinical research. 2017;23:5515-21. doi:10.12659/msm.905271.

17. Junhui $L$, Zhengbao $P$, Wenbin $X, L u H$, Shengyun $L$, Shunwu F, et al. Comparison of pedicle fixation by the Wiltse approach and the conventional posterior open approach for thoracolumbar fractures, using MRI, histological and electrophysiological analyses of the multifidus muscle. European spine journal: official publication of the European Spine Society, the European Spinal Deformity Society, and the European Section of the Cervical Spine. Research Society. 2017;26(5):1506-14. doi:10.1007/s00586-017-5010-1. 
18. Vaccaro AR, Oner C, Kepler CK, Dvorak M, Schnake K, Bellabarba C, et al. AOSpine thoracolumbar spine injury classification system: fracture description, neurological status, and key modifiers. Spine. 2013;38(23):2028-37. doi:10.1097/BRS.0b013e3182a8a381.

19. Gu G, Zhang H, He S, Cai X, Gu X, Jia J, et al. Percutaneous Pedicle Screw Placement in the Lumbar Spine: A Comparison Study Between the Novel Guidance System and the Conventional Fluoroscopy Method. J Spin Disord Tech. 2015;28(9):E522-7. doi:10.1097/BSD.0b013e3182aab222.

20. Ihedioha U, Sinha S, Campbell AC. Do creatine kinase (CK) levels influence the diagnosis or outcome in patients with compartment syndrome? Scot Med J. 2005;50(4):158-9. doi:10.1177/003693300505000407.

21. Gertzbein SD. Neurologic deterioration in patients with thoracic and lumbar fractures after admission to the hospital. Spine. 1994;19(15):1723-5. doi:10.1097/00007632-199408000-00011.

22. Thomas KC, Bailey CS, Dvorak MF, Kwon B, Fisher C. Comparison of operative and nonoperative treatment for thoracolumbar burst fractures in patients without neurological deficit: a systematic review. Journal of neurosurgery Spine. 2006;4(5):351-8. doi:10.3171/spi.2006.4.5.351.

23. Yi L, Jingping B, Gele J, Baoleri X, Taixiang W. Operative versus non-operative treatment for thoracolumbar burst fractures without neurological deficit. Cochrane Database Syst Rev. 2006(4):CD005079. doi:10.1002/14651858.CD005079.pub2.

24. Dai LY, Jiang SD, Wang XY, Jiang LS. A review of the management of thoracolumbar burst fractures. Surgical neurology. 2007;67(3):221-31. doi:10.1016/j.surneu.2006.08.081. discussion 31.

25. Gnanenthiran SR, Adie S, Harris IA. Nonoperative versus operative treatment for thoracolumbar burst fractures without neurologic deficit: a meta-analysis. Clin Orthop Relat Res. 2012;470(2):567-77. doi:10.1007/s11999-011-2157-7.

26. Kramer M, Katzmaier P, Eisele R, Ebert V, Kinzl L, Hartwig E. Surface electromyography-verified muscular damage associated with the open dorsal approach to the lumbar spine. European spine journal: official publication of the European Spine Society, the European Spinal Deformity Society, and the European Section of the Cervical Spine Research Society. 2001;10(5):414-20. doi:10.1007/s005860100294.

27. Wood KB, Li W, Lebl DR, Ploumis A. Management of thoracolumbar spine fractures. The spine journal: official journal of the North American Spine Society. 2014;14(1):145-64. doi:10.1016/j.spinee.2012.10.041.

28. Ohba T, Ebata S, Haro H. Comparison of serum markers for muscle damage, surgical blood loss, postoperative recovery, and surgical site pain after extreme lateral interbody fusion with percutaneous pedicle screws or traditional open posterior lumbar interbody fusion. BMC Musculoskelet Disord. 2017;18(1):415. doi:10.1186/s12891-017-1775-y.

29. Ringel F, Stoffel M, Stuer C, Meyer B. Minimally invasive transmuscular pedicle screw fixation of the thoracic and lumbar spine. Neurosurgery. 2006;59(4 Suppl 2):ONS361-6. doi:10.1227/01.NEU.0000223505.07815.74. discussion ONS6-7. 
30. Kreinest M, Rillig J, Kuffer M, Grutzner PA, Tinelli M, Matschke S. Comparison of pedicle screw misplacement following open vs. percutaneous dorsal instrumentation after traumatic spinal fracture. European journal of trauma emergency surgery: official publication of the European Trauma Society. 2019. doi:10.1007/s00068-019-01245-8.

31. Sun XY, Zhang XN, Hai Y. Percutaneous versus traditional and paraspinal posterior open approaches for treatment of thoracolumbar fractures without neurologic deficit: a meta-analysis. European spine journal: official publication of the European Spine Society, the European Spinal Deformity Society, and the European Section of the Cervical. Spine Research Society. 2017;26(5):1418-31. doi:10.1007/s00586-016-4818-4.

32. Lee JK, Jang JW, Kim TW, Kim TS, Kim SH, Moon SJ. Percutaneous short-segment pedicle screw placement without fusion in the treatment of thoracolumbar burst fractures: is it effective?: comparative study with open short-segment pedicle screw fixation with posterolateral fusion. Acta Neurochir. 2013;155(12):2305-12. doi:10.1007/s00701-013-1859-x. discussion 12.

\section{Figures}



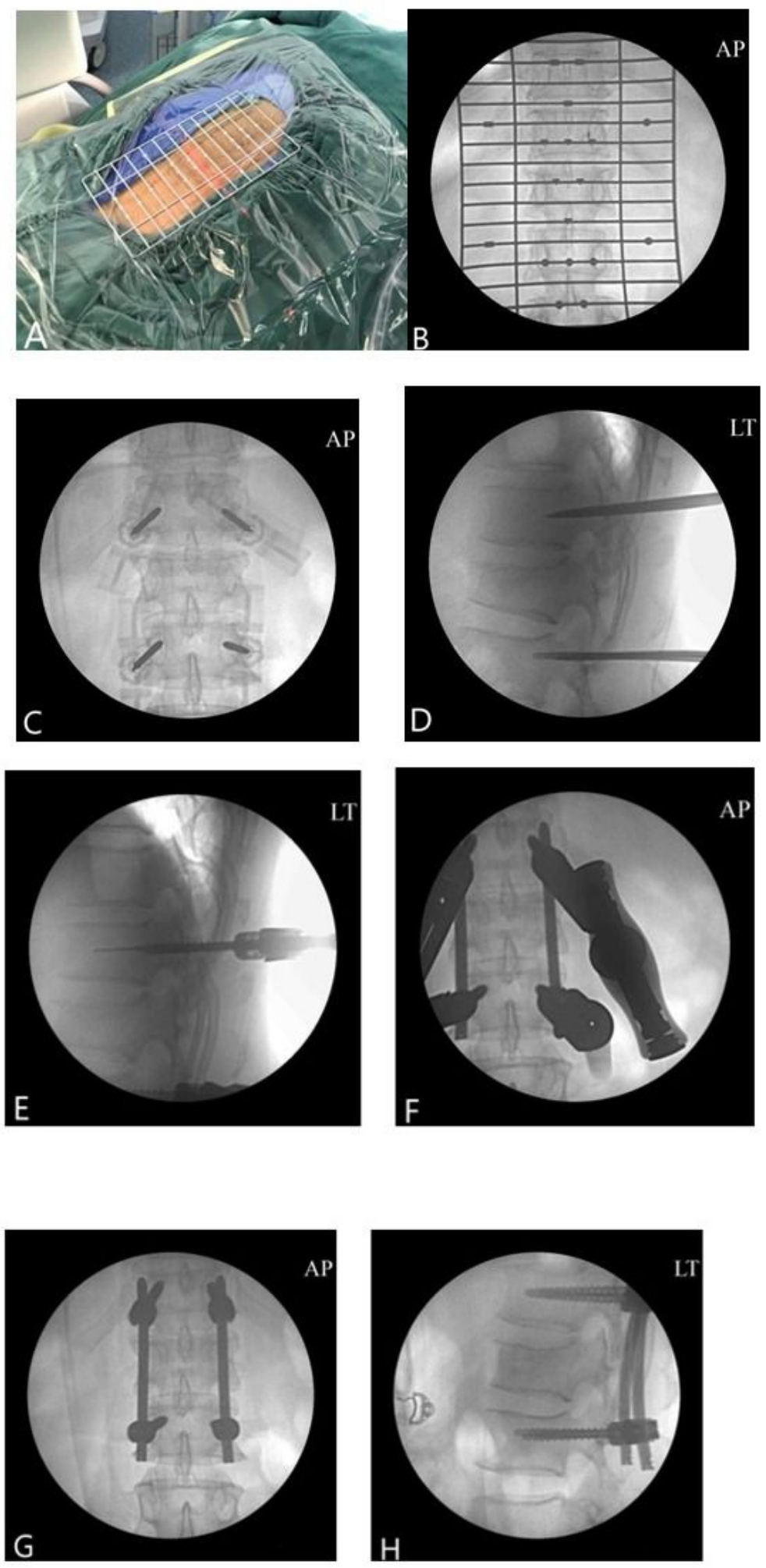

Figure 1

The process of the operation of percutaneous pedicle screw fixation (PPSF) A: The preoperative locator was placed onto the back of each of the patients with the central part. B: The correct pedicle projection and incision were obtained according to the different markers on the locator after observing the anteriorposterior fluoroscopic image. C \& D: The guide wire was inserted with the use of G-arm fluoroscopic 
image guidance. E: The pedicle screws were inserted along the guide wire. F: The rods were installed. G \& $H$ : Postoperative radiographical images: anterior-posterior view $(G)$ and lateral view $(H)$.

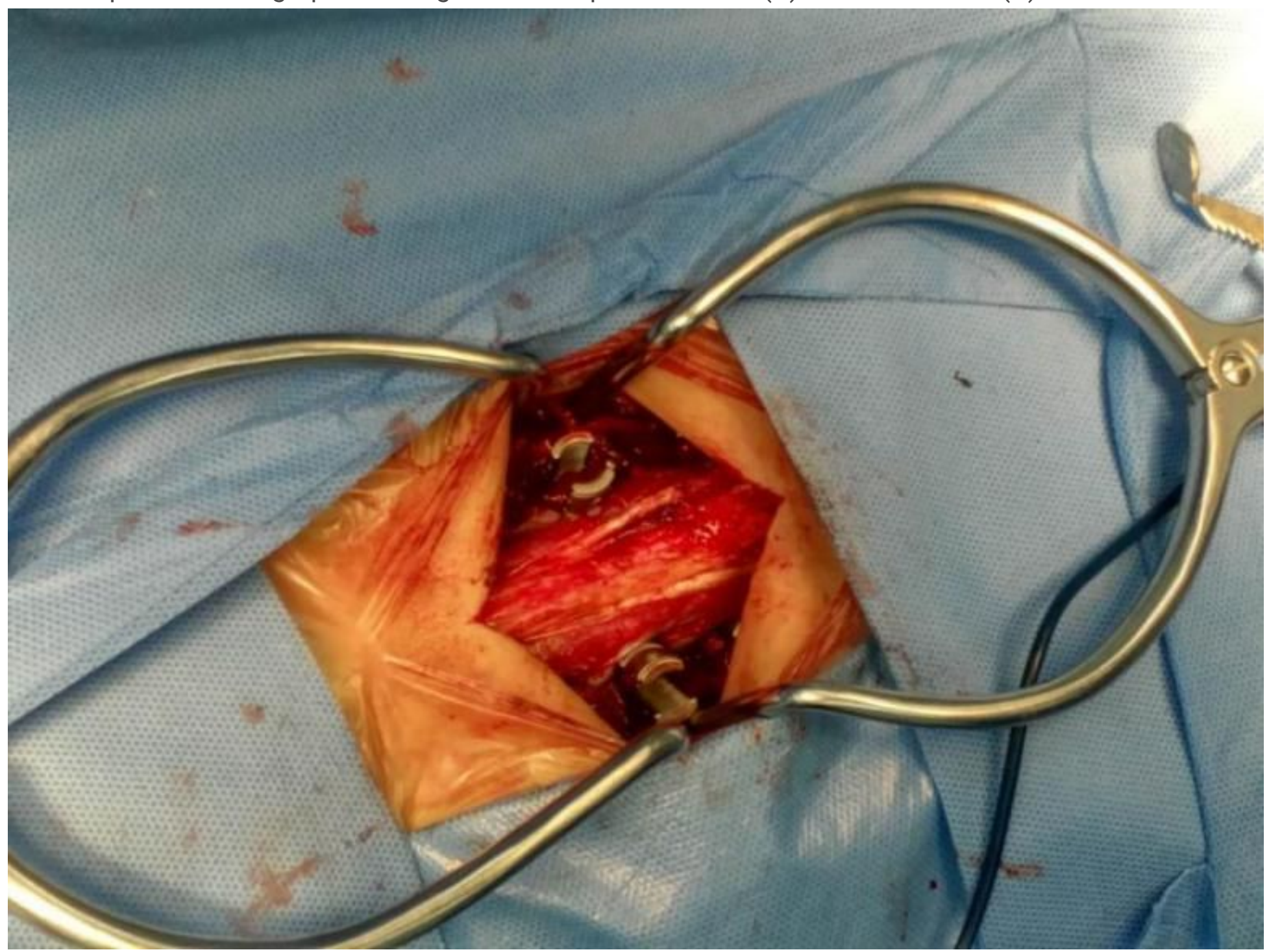

\section{Figure 2}

The paraspinal posterior open approach pedicle screw fixation was performed. An 8 to 10-cm posterior midline incision was performed in the target segment through the interspace between the multifidus and longissimus, in order for the pedicle entry point to be exposed. 